Acta Crystallographica Section E

Structure Reports

Online

ISSN 1600-5368

\section{(4-Bromophenyl)(3,6-dimethoxy-2- naphthyl)methanone}

\section{Yuichi Kato, Atsushi Nagasawa, Kotaro Kataoka, Akiko Okamoto and Noriyuki Yonezawa*}

Department of Organic and Polymer Materials Chemistry, Tokyo University of Agriculture \& Technology, 2-24-16 Naka-machi, Koganei, Tokyo 184-8588, Japan Correspondence e-mail: yonezawa@cc.tuat.ac.jp

Received 30 September 2010; accepted 7 October 2010

Key indicators: single-crystal X-ray study; $T=193 \mathrm{~K}$; mean $\sigma(\mathrm{C}-\mathrm{C})=0.003 \AA$; $R$ factor $=0.032 ; w R$ factor $=0.086 ;$ data-to-parameter ratio $=14.0$.

In the title compound, $\mathrm{C}_{19} \mathrm{H}_{15} \mathrm{BrO}_{3}$, the dihedral angle between the naphthalene ring system and the benzene ring is $62.51(8)^{\circ}$. The bridging carbonyl $\mathrm{C}-\mathrm{C}(=\mathrm{O})-\mathrm{C}$ plane makes dihedral angles of $47.07(6)^{\circ}$ with the naphthalene ring system and $24.20(10)^{\circ}$ with the benzene ring. A weak intermolecular $\mathrm{C}-\mathrm{H} \cdots \mathrm{O}$ hydrogen bond exists between the $\mathrm{H}$ atom of one methoxy group and the $\mathrm{O}$ atom of the other methoxy group in an adjacent molecule. The crystal packing is additionally stabilized by two types of weak intermolecular interactions involving the $\mathrm{Br}$ atom, $\mathrm{C}-\mathrm{H} \cdots \mathrm{Br}$ and $\mathrm{Br} \cdots \mathrm{O}$ [3.2802 (14) А].

\section{Related literature}

For electrophilic aromatic substitution of naphthalene derivatives affording peri-aroylated compounds regioselectively, see: Okamoto \& Yonezawa (2009). For the structures of closely related compounds, see: Kato et al. (2010); Muto et al. (2010); Nakaema et al. (2008); Watanabe et al. (2010a,b).<smiles>COc1ccc2cc(C(=O)c3ccc(Br)cc3)c(OC)cc2c1</smiles>

\section{Experimental}

Crystal data

$\mathrm{C}_{19} \mathrm{H}_{15} \mathrm{BrO}_{3}$

$M_{r}=371.22$

Monoclinic, $P 2_{1} / c$

$a=7.88917$ (14) §

$$
\begin{aligned}
& Z=4 \\
& \text { Cu } K \alpha \text { radiation } \\
& \mu=3.60 \mathrm{~mm}^{-1}
\end{aligned}
$$

Data collection

Rigaku R-AXIS RAPID

diffractometer

Absorption correction: numerical (NUMABS; Higashi, 1999)

$T_{\min }=0.161, T_{\max }=0.533$

Refinement

$R\left[F^{2}>2 \sigma\left(F^{2}\right)\right]=0.032$

$w R\left(F^{2}\right)=0.086$

$S=1.12$

2934 reflections
29541 measured reflections 2934 independent reflections 2767 reflections with $I>2 \sigma(I)$ $R_{\text {int }}=0.044$

Table 1

Hydrogen-bond geometry $\left(\AA{ }^{\circ}\right)$.

\begin{tabular}{lllll}
\hline$D-\mathrm{H} \cdots A$ & $D-\mathrm{H}$ & $\mathrm{H} \cdots A$ & $D \cdots A$ & $D-\mathrm{H} \cdots A$ \\
\hline $\mathrm{C} 19-\mathrm{H} 19 A \cdots \mathrm{O} 1^{\mathrm{i}}$ & 0.96 & 2.53 & $3.477(2)$ & 170 \\
$\mathrm{C} 5-\mathrm{H} 5 \cdots \mathrm{Br}^{\mathrm{ii}}$ & 0.93 & 2.98 & $3.8441(18)$ & 155 \\
\hline
\end{tabular}

Symmetry codes: (i) $x+1, y, z+1$; (ii) $-x,-y,-z$.

Data collection: PROCESS-AUTO (Rigaku, 1998); cell refinement: PROCESS-AUTO; data reduction: CrystalStructure (Rigaku/ MSC, 2004); program(s) used to solve structure: SIR2004 (Burla et al., 2005); $\operatorname{program}(\mathrm{s})$ used to refine structure: SHELXL97 (Sheldrick, 2008); molecular graphics: ORTEPIII (Burnett \& Johnson, 1996); software used to prepare material for publication: SHELXL97.

The authors would express their gratitude to Professor Keiichi Noguchi, Instrumentation Analysis Center, Tokyo University of Agriculture \& Technology, for technical advice. This work was partially supported by the Sasagawa Scientific Research Grant from the Japan Science Society.

Supplementary data and figures for this paper are available from the IUCr electronic archives (Reference: VM2049).

\section{References}

Burla, M. C., Caliandro, R., Camalli, M., Carrozzini, B., Cascarano, G. L., De Caro, L., Giacovazzo, C., Polidori, G. \& Spagna, R. (2005). J. Appl. Cryst. 38, 381-388.

Burnett, M. N. \& Johnson, C. K. (1996). ORTEPIII. Report ORNL-6895. Oak Ridge National Laboratory. Tennessee, USA.

Higashi, T. (1999). NUMABS. Rigaku Corporation, Tokyo, Japan.

Kato, Y., Nagasawa, A., Hijikata, D., Okamoto, A. \& Yonezawa, N. (2010). Acta Cryst. E66, o2659.

Muto, T., Kato, Y., Nagasawa, A., Okamoto, A. \& Yonezawa, N. (2010). Acta Cryst. E66, o2752.

Nakaema, K., Okamoto, A., Imaizumi, M., Noguchi, K. \& Yonezawa, N. (2008). Acta Cryst. E64, o612.

Okamoto, A. \& Yonezawa, N. (2009). Chem. Lett. 38, 914-915.

Rigaku (1998). PROCESS-AUTO. Rigaku Corporation, Tokyo, Japan.

Rigaku/MSC (2004). CrystalStructure. Rigaku/MSC, The Woodlands, Texas, USA.

Sheldrick, G. M. (2008). Acta Cryst. A64, 112-122.

Watanabe, S., Muto, T., Nagasawa, A., Okamoto, A. \& Yonezawa, N. (2010b). Acta Cryst. E66, o712.

Watanabe, S., Nakaema, K., Muto, T., Okamoto, A. \& Yonezawa, N. (2010a). Acta Cryst. E66, o403. 


\section{supporting information}

Acta Cryst. (2010). E66, o2795 [https://doi.org/10.1107/S160053681004016X]

\section{(4-Bromophenyl)(3,6-dimethoxy-2-naphthyl)methanone}

\section{Yuichi Kato, Atsushi Nagasawa, Kotaro Kataoka, Akiko Okamoto and Noriyuki Yonezawa}

\section{S1. Comment}

In the course of our study on selective electrophilic aromatic aroylation of 2,7-dimethoxynaphthalene, peri-aroylnaphthalene compounds have proved to be formed regioselectively with the aid of suitable acidic mediator (Okamoto \& Yonezawa, 2009). Recently, we reported the structures of 1,8-diaroyl-2,7-dimethoxynaphthalenes, i. e., 1,8-bis(4-methylbenzoyl)-2,7-dimethoxynaphthalene (Muto et al., 2010), bis(4-bromophenyl)(2,7-dimethoxynaphthalene-1,8-diyl)dimethanone (Watanabe et al., 2010a), and 1-aroyl-2,7-dimethoxynaphthalene, i. e., 1-benzoyl-2,7-dimethoxynaphthalene (Kato et al., 2010). The aroyl groups at the 1,8-positions of the naphthalene rings in these compounds are twistedly bonded in a perpendicular manner but the benzene ring moieties of the aroyl groups tilt slightly toward the exo sides of the naphthalene rings. 1-Aroyl homologues also revealed essentially the same non-coplanar structure as observed for 1,8diaroylated naphthalenes.

Furthermore, we also reported the crystal structure analysis of the corresponding $\beta$-isomers of 3-aroyl-2,7-dimethoxynaphthalenes such as 2-(4-chlorobenzoyl)-3,6-dimethoxynaphthalene (Nakaema et al., 2008) and (3,6-dimethoxy-2naphthyl)(4-fluorophenyl)methanone (Watanabe et al., 2010b). In these 3-aroylated naphthalenes, which are generally regarded to be thermodynamically more stable than the corresponding 1-positioned isomeric molecules, the aroyl groups are shown connected to the naphthalene rings in a moderately twisted fashion. As part of our ongoing study on these homologous molecules, the synthesis and crystal structure of the title compound, a 3-monoaroylnaphthalene bearing a bromo group, is discussed in this article. The title compound was prepared by a direct condensation reaction of 2,7-dimethoxynaphthalene with 4-bromobenzoic acid.

The molecular structure of the title molecule is displayed in Fig. 1. The 4-bromophenyl group is bonded twistedly away from the attached naphthalene ring. The dihedral angle between the best planes of the bromophenyl ring $(\mathrm{C} 12-\mathrm{C} 17)$ and the naphthalene ring $(\mathrm{C} 1-\mathrm{C} 10)$ is $62.51(8)^{\circ}$. The bridging carbonyl plane $(\mathrm{O} 3-\mathrm{C} 6-\mathrm{C} 11-\mathrm{C} 12)$ makes a relatively large dihedral angle of $47.07(9)^{\circ}$ with the naphthalene ring $(\mathrm{C} 1-\mathrm{C} 10)\left[\mathrm{C} 5-\mathrm{C} 6-\mathrm{C} 11-\mathrm{O} 3\right.$ torsion angle $=46.0(2)^{\circ}$, whereas it makes a rather small angle of $24.20(10)^{\circ}$ with 4-bromophenyl ring $(\mathrm{C} 12-\mathrm{C} 17)[\mathrm{O} 3-\mathrm{C} 11-\mathrm{C} 12-\mathrm{C} 17$ torsion angle $\left.=24.1(3)^{\circ}\right]$.

In the crystal structure, the molecular packing of the title compound is stabilized mainly by van der Waals interactions. Moreover, there is a $\mathrm{C}-\mathrm{H} \cdots \mathrm{O}$ hydrogen bond between a hydrogen of the 2-methoxy group, which is situated adjacent to the bromophenyl group, and the ethereal oxygen atom of the 7-methoxy group in the neighboring molecule (Table 1, Fig. 2).

The crystal packing is additionally stabilized by two types of weak intermolecular interactions with the bromine atom: $\mathrm{Br} 1 \cdots \mathrm{O} 3^{\mathrm{i}}=3.2802$ (14) $\AA$, and $\mathrm{Br} 1 \cdots \mathrm{H} 5^{\mathrm{ii}}=2.98 \AA$ [symmetry operations: (i) $x, y, z+1$, (ii) $-x,-y,-z$ ] (Fig. 3 ) . 


\section{S2. Experimental}

The title compound was prepared by treatment of a mixture of 2,7-dimethoxynaphthalene (1.88 g, $10 \mathrm{mmol})$ and 4bromobenzoic acid (2.02 g, $10 \mathrm{mmol})$ with phosphorus pentoxide-methanesulfonic acid mixture $\left(\mathrm{P}_{2} \mathrm{O}_{5}-\mathrm{MsOH}[1 / 10\right.$ $\mathrm{w} / \mathrm{w}] ; 10 \mathrm{~mL}$ ). After the reaction mixture was stirred at $353 \mathrm{~K}$ for 8 hours, the mixture was poured into ice-cooled water and extracted with $\mathrm{CHCl}_{3}(10 \mathrm{ml} \times 3)$. The combined extracts were washed with $2 \mathrm{M}$ aqueous $\mathrm{NaOH}$ followed by washing with brine. The organic layer thus obtained was dried over anhydrous $\mathrm{MgSO}_{4}$. The solvent was removed under reduced pressure to give a cake (yield $3.07 \mathrm{~g}, 83 \%$ ). The crude product was purified by flush silica gel chromatography $\left(\mathrm{CHCl}_{3}\right)$. Colorless platelet single crystals suitable for X-ray diffraction were obtained by crystallization from ethanol and chloroform.

Spectroscopic Data:

${ }^{1} \mathrm{H}$ NMR (300 MHz, CDCl $) \delta 3.81(3 \mathrm{H}, \mathrm{s}), 3.93(3 \mathrm{H}, \mathrm{s}), 7.03(1 \mathrm{H}, \mathrm{dd}, 9.0 \mathrm{~Hz}), 7.09(1 \mathrm{H}, \mathrm{d}, \mathrm{J}=2.4 \mathrm{~Hz}), 7.12(1 \mathrm{H}, \mathrm{s})$, $7.56(2 \mathrm{H}, \mathrm{d}, \mathrm{J}=8.4 \mathrm{~Hz}), 7.67-7.71(3 \mathrm{H}, \mathrm{m}), 7.78(1 \mathrm{H}, \mathrm{s})$.

${ }^{13} \mathrm{C}$ NMR $\left(75 \mathrm{MHz}, \mathrm{CDCl}_{3}\right) \delta 55.32,55.49,105.01,105.42,117.16,123.18,127.32,127.92,130.05,130.25,131.30$, 131.46, 136.96, 137.28, 155.64, 159.45, 194.94 .

IR (KBr): 1626, 1585, 1501, $1134 \mathrm{~cm}^{-1}$.

HRMS (m/z): $[M+\mathrm{H}]^{+}$calcd for $\mathrm{C}_{19} \mathrm{H}_{16} \mathrm{BrO}_{3}, 371.0283$; found, 371.0298 .

\section{S3. Refinement}

All $\mathrm{H}$ atoms were found in a difference map and were subsequently refined as riding atoms, with $\mathrm{C}-\mathrm{H}=0.93$ (aromatic) and 0.96 (methyl) $\AA$, and with $U_{\text {iso }}(\mathrm{H})=1.2 U_{\mathrm{eq}}(\mathrm{C})$.

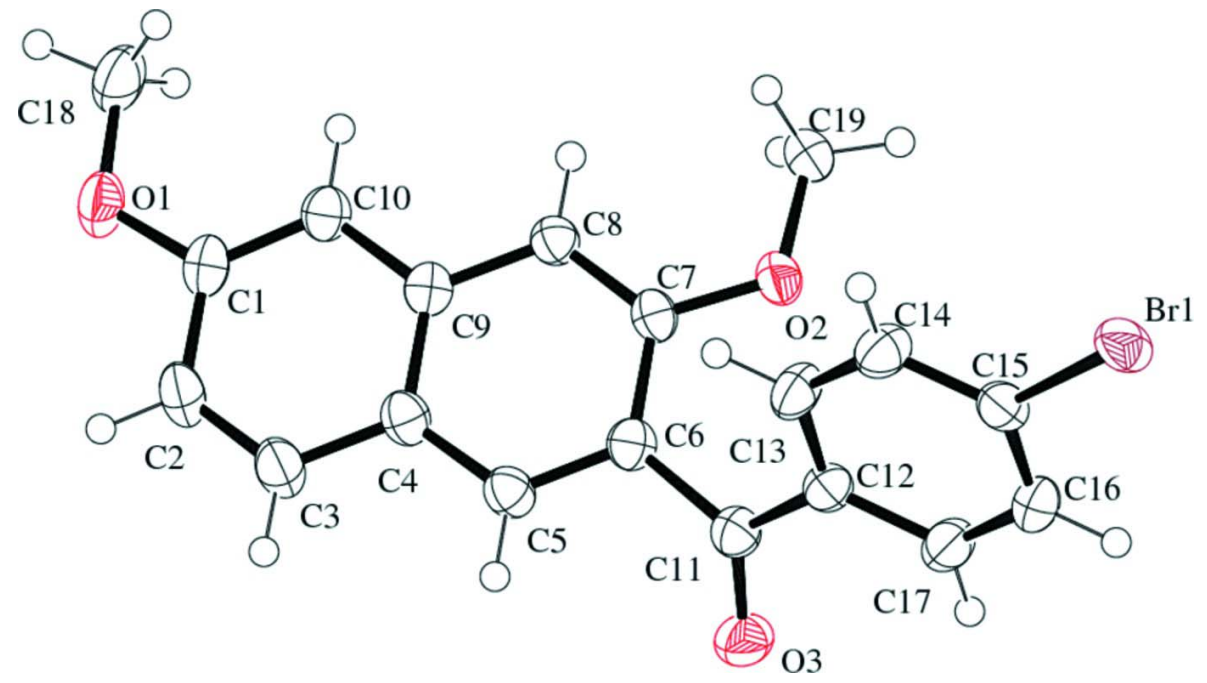

Figure 1

Molecular structure with displacement ellipsoids at 50\% probability for non- $\mathrm{H}$ atoms. 


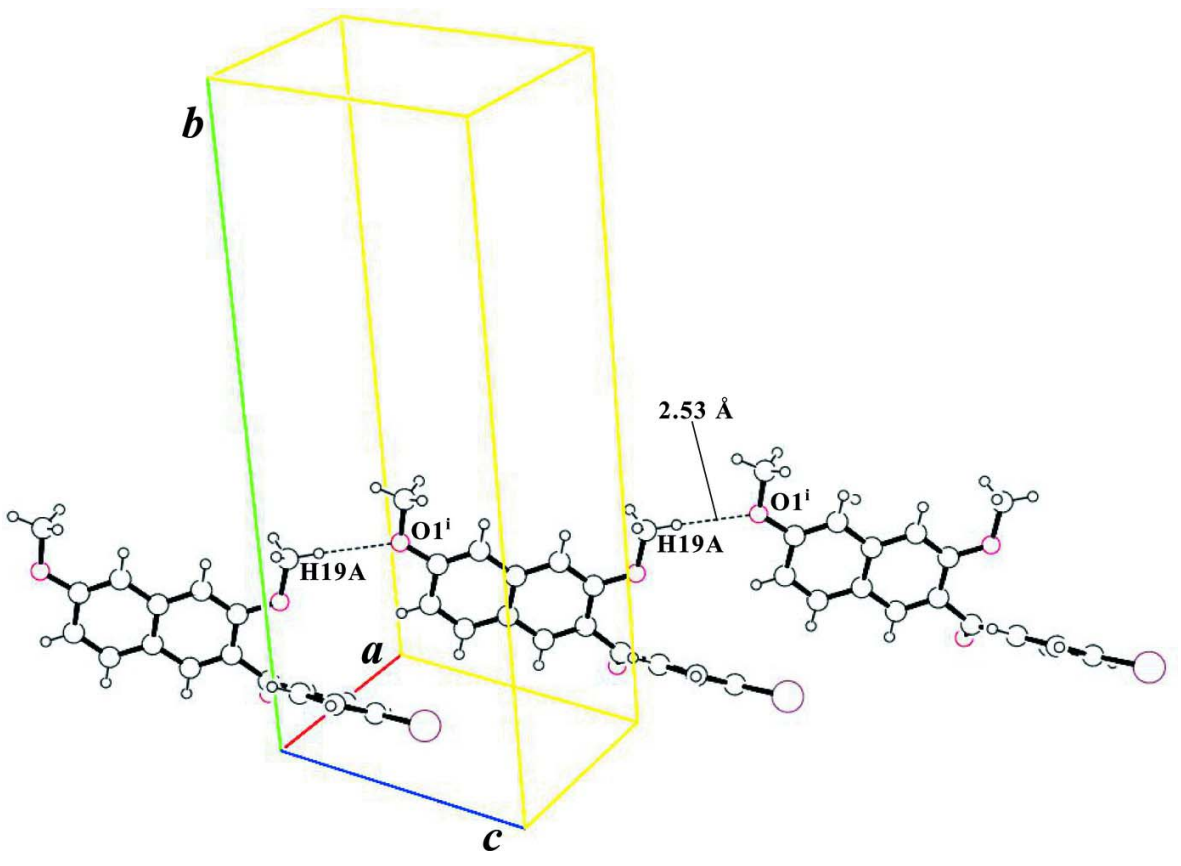

Figure 2

$\mathrm{C}-\mathrm{H} \cdots \mathrm{O}$ interactions (dotted lines) [symmetry code: (i) $x+1, y, z+1$ ].

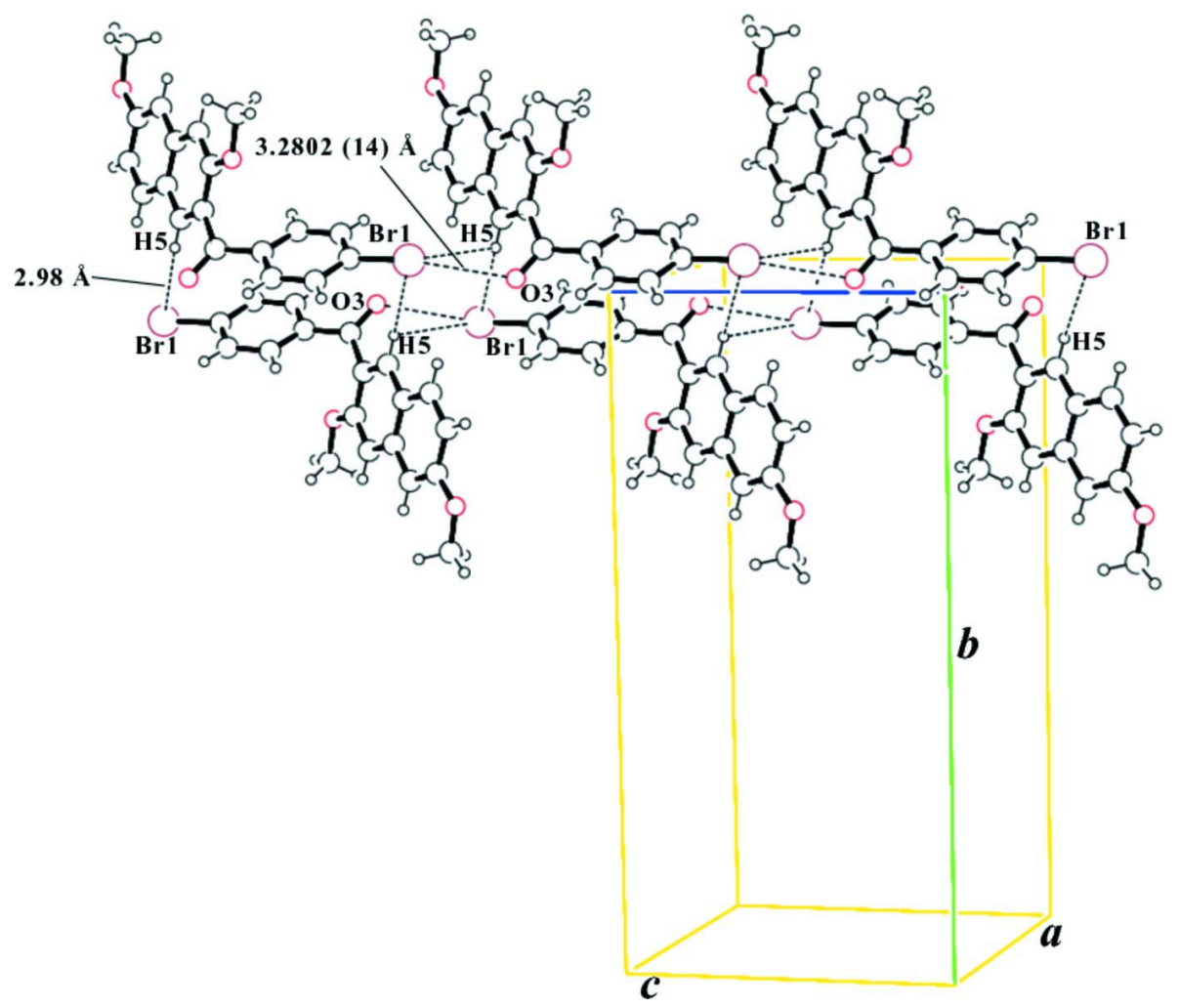

Figure 3

Two types of intermolecular weak interactions with bromine atom $\mathrm{Br} 1$ (dotted lines). 
(4-Bromophenyl)(3,6-dimethoxy-2-naphthyl)methanone

Crystal data

$\mathrm{C}_{19} \mathrm{H}_{15} \mathrm{BrO}_{3}$

$M_{r}=371.22$

Monoclinic, $P 2_{1} / c$

Hall symbol: -P $2 \mathrm{ybc}$

$a=7.88917$ (14) $\AA$

$b=21.0182(4) \AA$

$c=10.06272$ (18) $\AA$

$\beta=105.971(1)^{\circ}$

$V=1604.16(5) \AA^{3}$

$Z=4$

\section{Data collection}

Rigaku R-AXIS RAPID diffractometer

Radiation source: rotating anode

Graphite monochromator

Detector resolution: 10.00 pixels $\mathrm{mm}^{-1}$

$\omega$ scans

Absorption correction: numerical

(NUMABS; Higashi, 1999)

$T_{\text {min }}=0.161, T_{\max }=0.533$

\section{Refinement}

Refinement on $F^{2}$

Least-squares matrix: full

$R\left[F^{2}>2 \sigma\left(F^{2}\right)\right]=0.032$

$w R\left(F^{2}\right)=0.086$

$S=1.12$

2934 reflections

210 parameters

0 restraints

Primary atom site location: structure-invariant

direct methods
$F(000)=752$

$D_{\mathrm{x}}=1.537 \mathrm{Mg} \mathrm{m}^{-3}$

Melting point $=416.9-419.5 \mathrm{~K}$

$\mathrm{Cu} K \alpha$ radiation, $\lambda=1.54187 \AA$

Cell parameters from 26572 reflections

$\theta=4.2-68.3^{\circ}$

$\mu=3.60 \mathrm{~mm}^{-1}$

$T=193 \mathrm{~K}$

Platelet, colorless

$0.60 \times 0.40 \times 0.20 \mathrm{~mm}$

29541 measured reflections

2934 independent reflections

2767 reflections with $I>2 \sigma(I)$

$R_{\text {int }}=0.044$

$\theta_{\max }=68.3^{\circ}, \theta_{\min }=4.2^{\circ}$

$h=-9 \rightarrow 9$

$k=-25 \rightarrow 25$

$l=-12 \rightarrow 12$

Secondary atom site location: difference Fourier map

Hydrogen site location: inferred from neighbouring sites

$\mathrm{H}$-atom parameters constrained

$w=1 /\left[\sigma^{2}\left(F_{\mathrm{o}}^{2}\right)+(0.0497 P)^{2}+0.6344 P\right]$

where $P=\left(F_{\mathrm{o}}^{2}+2 F_{\mathrm{c}}^{2}\right) / 3$

$(\Delta / \sigma)_{\max }<0.001$

$\Delta \rho_{\max }=0.43$ e $\AA^{-3}$

$\Delta \rho_{\min }=-1.03$ e $\AA^{-3}$

\section{Special details}

Geometry. All e.s.d.'s (except the e.s.d. in the dihedral angle between two 1.s. planes) are estimated using the full covariance matrix. The cell e.s.d.'s are taken into account individually in the estimation of e.s.d.'s in distances, angles and torsion angles; correlations between e.s.d.'s in cell parameters are only used when they are defined by crystal symmetry. An approximate (isotropic) treatment of cell e.s.d.'s is used for estimating e.s.d.'s involving 1.s. planes.

Refinement. Refinement of $F^{2}$ against ALL reflections. The weighted $R$-factor $w R$ and goodness of fit $S$ are based on $F^{2}$, conventional $R$-factors $R$ are based on $F$, with $F$ set to zero for negative $F^{2}$. The threshold expression of $F^{2}>\sigma\left(F^{2}\right)$ is used only for calculating $R$-factors(gt) etc. and is not relevant to the choice of reflections for refinement. $R$-factors based on $F^{2}$ are statistically about twice as large as those based on $F$, and $R$-factors based on ALL data will be even larger.

Fractional atomic coordinates and isotropic or equivalent isotropic displacement parameters $\left(\AA^{2}\right)$

\begin{tabular}{lllll}
\hline & $x$ & $y$ & $z$ & $U_{\text {iso }} * / U_{\text {eq }}$ \\
\hline Br1 & $0.24719(3)$ & $0.056869(10)$ & $0.494126(19)$ & $0.03961(11)$ \\
O1 & $-0.46425(17)$ & $0.28009(7)$ & $-0.69867(14)$ & $0.0406(3)$ \\
O2 & $0.20106(17)$ & $0.19970(6)$ & $-0.05734(13)$ & $0.0342(3)$
\end{tabular}




$\begin{array}{lllll}\text { O3 } & 0.24997(19) & 0.03129(7) & -0.18371(14) & 0.0405(3) \\ \text { C1 } & -0.3611(2) & 0.24042(10) & -0.6033(2) & 0.0324(4) \\ \text { C2 } & -0.3738(3) & 0.17558(10) & -0.6441(2) & 0.0384(4) \\ \text { H2 } & -0.4485 & 0.1636 & -0.7292 & 0.046^{*} \\ \text { C3 } & -0.2762(3) & 0.13090(10) & -0.5582(2) & 0.0375(4) \\ \text { H3 } & -0.2853 & 0.0885 & -0.5854 & 0.045^{*} \\ \text { C4 } & -0.1608(2) & 0.14784(9) & -0.4282(2) & 0.0302(4) \\ \text { C5 } & -0.0537(2) & 0.10319(9) & -0.33881(19) & 0.0303(4) \\ \text { H5 } & -0.0616 & 0.0605 & -0.3643 & 0.036^{*} \\ \text { C6 } & 0.0620(2) & 0.12056(9) & -0.21526(18) & 0.0284(4) \\ \text { C7 } & 0.0759(2) & 0.18618(9) & -0.17702(18) & 0.0279(4) \\ \text { C8 } & -0.0283(2) & 0.23056(9) & -0.26096(19) & 0.0284(4) \\ \text { H8 } & -0.0198 & 0.2730 & -0.2340 & 0.034^{*} \\ \text { C9 } & -0.1488(2) & 0.21296(9) & -0.38808(19) & 0.0278(4) \\ \text { C10 } & -0.2524(2) & 0.25890(10) & -0.47798(19) & 0.0303(4) \\ \text { H10 } & -0.2466 & 0.3015 & -0.4520 & 0.036^{*} \\ \text { C11 } & 0.1740(2) & 0.06982(9) & -0.1299(2) & 0.0308(4) \\ \text { C12 } & 0.1884(2) & 0.06594(8) & 0.02077(19) & 0.0291(4) \\ \text { C13 } & 0.0583(2) & 0.09052(9) & 0.0753(2) & 0.0349(4) \\ \text { H13 } & -0.0401 & 0.1100 & 0.0169 & 0.042^{*} \\ \text { C14 } & 0.0731(3) & 0.08652(9) & 0.2154(2) & 0.0363(4) \\ \text { H14 } & -0.0155 & 0.1024 & 0.2509 & 0.044^{*} \\ \text { C15 } & 0.2217(3) & 0.05857(8) & 0.3014(2) & 0.0312(4) \\ \text { C16 } & 0.3515(3) & 0.03239(10) & 0.2499(2) & 0.0370(4) \\ \text { H16 } & 0.4493 & 0.0127 & 0.3087 & 0.044^{*} \\ \text { C17 } & 0.3339(3) & 0.03590(10) & 0.1099(2) & 0.0358(4) \\ \text { H17 } & 0.4200 & 0.0180 & 0.0743 & 0.043^{*} \\ \text { C18 } & -0.4465(3) & 0.34656(10) & -0.6723(2) & 0.0454(5) \\ \text { H18A } & -0.5287 & 0.3692 & -0.7451 & 0.055^{*} \\ \text { H18B } & -0.3286 & 0.3596 & -0.6683 & 0.055^{*} \\ \text { H18C } & -0.4704 & 0.3558 & -0.5857 & 0.055^{*} \\ \text { C19 } & 0.2347(2) & 0.26549(10) & -0.0224(2) & 0.0348(4) \\ \text { H19A } & 0.3294 & 0.2689 & 0.0609 & 0.042^{*} \\ \text { H19B } & 0.1305 & 0.2847 & -0.0086 & 0.042^{*} \\ \text { H19C } & 0.2667 & 0.2869 & -0.0962 & 0.042^{*} \\ & & & & \end{array}$

Atomic displacement parameters $\left(\AA^{2}\right)$

\begin{tabular}{lllllll}
\hline & $U^{11}$ & $U^{22}$ & $U^{33}$ & $U^{12}$ & $U^{13}$ & $U^{23}$ \\
\hline Br1 & $0.05907(18)$ & $0.03269(17)$ & $0.02418(16)$ & $0.00179(8)$ & $0.00659(11)$ & $-0.00036(7)$ \\
O1 & $0.0367(7)$ & $0.0454(8)$ & $0.0321(7)$ & $0.0022(6)$ & $-0.0032(6)$ & $0.0083(6)$ \\
O2 & $0.0389(7)$ & $0.0309(7)$ & $0.0260(7)$ & $0.0006(5)$ & $-0.0024(5)$ & $-0.0014(5)$ \\
O3 & $0.0488(8)$ & $0.0407(8)$ & $0.0321(7)$ & $0.0134(6)$ & $0.0113(6)$ & $-0.0007(6)$ \\
C1 & $0.0269(8)$ & $0.0411(11)$ & $0.0274(9)$ & $0.0000(7)$ & $0.0044(7)$ & $0.0071(8)$ \\
C2 & $0.0370(9)$ & $0.0451(12)$ & $0.0269(10)$ & $-0.0061(8)$ & $-0.0017(8)$ & $0.0000(8)$ \\
C3 & $0.0399(10)$ & $0.0365(11)$ & $0.0309(11)$ & $-0.0069(8)$ & $0.0011(8)$ & $-0.0009(9)$ \\
C4 & $0.0301(8)$ & $0.0321(10)$ & $0.0278(9)$ & $-0.0050(7)$ & $0.0071(7)$ & $0.0003(7)$ \\
C5 & $0.0353(9)$ & $0.0280(9)$ & $0.0272(9)$ & $-0.0032(7)$ & $0.0077(7)$ & $-0.0001(7)$
\end{tabular}


supporting information

\begin{tabular}{lllllll} 
C6 & $0.0310(8)$ & $0.0298(9)$ & $0.0248(9)$ & $0.0001(7)$ & $0.0083(7)$ & $0.0030(7)$ \\
C7 & $0.0287(8)$ & $0.0325(9)$ & $0.0216(8)$ & $-0.0019(7)$ & $0.0055(6)$ & $-0.0005(7)$ \\
C8 & $0.0308(8)$ & $0.0271(9)$ & $0.0263(9)$ & $-0.0003(7)$ & $0.0066(7)$ & $-0.0001(7)$ \\
C9 & $0.0261(8)$ & $0.0320(10)$ & $0.0260(9)$ & $-0.0010(7)$ & $0.0083(7)$ & $0.0028(7)$ \\
C10 & $0.0298(9)$ & $0.0326(10)$ & $0.0282(9)$ & $-0.0004(7)$ & $0.0074(7)$ & $0.0036(7)$ \\
C11 & $0.0324(9)$ & $0.0292(9)$ & $0.0292(10)$ & $0.0004(7)$ & $0.0060(7)$ & $0.0010(8)$ \\
C12 & $0.0331(9)$ & $0.0248(9)$ & $0.0282(10)$ & $0.0013(7)$ & $0.0063(7)$ & $0.0023(7)$ \\
C13 & $0.0339(9)$ & $0.0383(10)$ & $0.0311(10)$ & $0.0102(8)$ & $0.0067(8)$ & $0.0084(8)$ \\
C14 & $0.0404(10)$ & $0.0361(10)$ & $0.0337(10)$ & $0.0087(8)$ & $0.0123(8)$ & $0.0054(8)$ \\
C15 & $0.0427(10)$ & $0.0243(9)$ & $0.0246(9)$ & $-0.0018(7)$ & $0.0059(8)$ & $0.0015(7)$ \\
C16 & $0.0371(9)$ & $0.0375(11)$ & $0.0313(10)$ & $0.0092(8)$ & $0.0006(8)$ & $0.0032(8)$ \\
C17 & $0.0358(9)$ & $0.0372(11)$ & $0.0335(10)$ & $0.0107(8)$ & $0.0081(8)$ & $0.0030(9)$ \\
C18 & $0.0429(11)$ & $0.0452(12)$ & $0.0415(12)$ & $0.0071(9)$ & $0.0004(9)$ & $0.0127(10)$ \\
C19 & $0.0362(9)$ & $0.0335(11)$ & $0.0304(9)$ & $-0.0010(7)$ & $0.0019(8)$ & $-0.0052(8)$ \\
& & & & & & \\
\hline
\end{tabular}

Geometric parameters $\left(\AA,{ }^{\circ}\right)$

\begin{tabular}{|c|c|c|c|}
\hline $\mathrm{Br} 1-\mathrm{C} 15$ & $1.8942(19)$ & $\mathrm{C} 8-\mathrm{H} 8$ & 0.9300 \\
\hline $\mathrm{O} 1-\mathrm{C} 1$ & $1.359(2)$ & $\mathrm{C} 9-\mathrm{C} 10$ & $1.418(3)$ \\
\hline $\mathrm{O} 1-\mathrm{C} 18$ & $1.422(3)$ & $\mathrm{C} 10-\mathrm{H} 10$ & 0.9300 \\
\hline $\mathrm{O} 2-\mathrm{C} 7$ & $1.361(2)$ & $\mathrm{C} 11-\mathrm{C} 12$ & $1.491(3)$ \\
\hline $\mathrm{O} 2-\mathrm{C} 19$ & $1.433(2)$ & $\mathrm{C} 12-\mathrm{C} 13$ & $1.390(3)$ \\
\hline $\mathrm{O} 3-\mathrm{C} 11$ & $1.219(2)$ & $\mathrm{C} 12-\mathrm{C} 17$ & $1.398(3)$ \\
\hline $\mathrm{C} 1-\mathrm{C} 10$ & $1.372(3)$ & $\mathrm{C} 13-\mathrm{C} 14$ & $1.384(3)$ \\
\hline $\mathrm{C} 1-\mathrm{C} 2$ & $1.419(3)$ & $\mathrm{C} 13-\mathrm{H} 13$ & 0.9300 \\
\hline $\mathrm{C} 2-\mathrm{C} 3$ & $1.362(3)$ & $\mathrm{C} 14-\mathrm{C} 15$ & $1.383(3)$ \\
\hline $\mathrm{C} 2-\mathrm{H} 2$ & 0.9300 & $\mathrm{C} 14-\mathrm{H} 14$ & 0.9300 \\
\hline $\mathrm{C} 3-\mathrm{C} 4$ & $1.419(3)$ & $\mathrm{C} 15-\mathrm{C} 16$ & $1.383(3)$ \\
\hline $\mathrm{C} 3-\mathrm{H} 3$ & 0.9300 & $\mathrm{C} 16-\mathrm{C} 17$ & $1.379(3)$ \\
\hline $\mathrm{C} 4-\mathrm{C} 5$ & $1.409(3)$ & $\mathrm{C} 16-\mathrm{H} 16$ & 0.9300 \\
\hline $\mathrm{C} 4-\mathrm{C} 9$ & $1.423(3)$ & C17-H17 & 0.9300 \\
\hline $\mathrm{C} 5-\mathrm{C} 6$ & $1.374(3)$ & $\mathrm{C} 18-\mathrm{H} 18 \mathrm{~A}$ & 0.9600 \\
\hline $\mathrm{C} 5-\mathrm{H} 5$ & 0.9300 & $\mathrm{C} 18-\mathrm{H} 18 \mathrm{~B}$ & 0.9600 \\
\hline $\mathrm{C} 6-\mathrm{C} 7$ & $1.428(3)$ & $\mathrm{C} 18-\mathrm{H} 18 \mathrm{C}$ & 0.9600 \\
\hline $\mathrm{C} 6-\mathrm{C} 11$ & $1.496(3)$ & $\mathrm{C} 19-\mathrm{H} 19 \mathrm{~A}$ & 0.9600 \\
\hline $\mathrm{C} 7-\mathrm{C} 8$ & $1.370(3)$ & C19-H19B & 0.9600 \\
\hline $\mathrm{C} 8-\mathrm{C} 9$ & $1.417(2)$ & $\mathrm{C} 19-\mathrm{H} 19 \mathrm{C}$ & 0.9600 \\
\hline $\mathrm{C} 1-\mathrm{O} 1-\mathrm{C} 18$ & $117.51(15)$ & $\mathrm{O} 3-\mathrm{C} 11-\mathrm{C} 6$ & $120.23(18)$ \\
\hline $\mathrm{C} 7-\mathrm{O} 2-\mathrm{C} 19$ & $117.30(14)$ & $\mathrm{C} 12-\mathrm{C} 11-\mathrm{C} 6$ & $119.37(16)$ \\
\hline $\mathrm{O} 1-\mathrm{C} 1-\mathrm{C} 10$ & $125.22(19)$ & $\mathrm{C} 13-\mathrm{C} 12-\mathrm{C} 17$ & $118.65(18)$ \\
\hline $\mathrm{O} 1-\mathrm{C} 1-\mathrm{C} 2$ & $113.82(17)$ & $\mathrm{C} 13-\mathrm{C} 12-\mathrm{C} 11$ & $121.55(16)$ \\
\hline $\mathrm{C} 10-\mathrm{C} 1-\mathrm{C} 2$ & $120.96(17)$ & $\mathrm{C} 17-\mathrm{C} 12-\mathrm{C} 11$ & $119.78(17)$ \\
\hline $\mathrm{C} 3-\mathrm{C} 2-\mathrm{C} 1$ & $119.73(18)$ & $\mathrm{C} 14-\mathrm{C} 13-\mathrm{C} 12$ & $121.02(17)$ \\
\hline $\mathrm{C} 3-\mathrm{C} 2-\mathrm{H} 2$ & 120.1 & $\mathrm{C} 14-\mathrm{C} 13-\mathrm{H} 13$ & 119.5 \\
\hline $\mathrm{C} 1-\mathrm{C} 2-\mathrm{H} 2$ & 120.1 & $\mathrm{C} 12-\mathrm{C} 13-\mathrm{H} 13$ & 119.5 \\
\hline $\mathrm{C} 2-\mathrm{C} 3-\mathrm{C} 4$ & $121.30(19)$ & $\mathrm{C} 15-\mathrm{C} 14-\mathrm{C} 13$ & $118.84(18)$ \\
\hline $\mathrm{C} 2-\mathrm{C} 3-\mathrm{H} 3$ & 119.4 & C15-C14-H14 & 120.6 \\
\hline
\end{tabular}




$$
\begin{aligned}
& \mathrm{C} 4-\mathrm{C} 3-\mathrm{H} 3 \\
& \mathrm{C} 5-\mathrm{C} 4-\mathrm{C} 3 \\
& \mathrm{C} 5-\mathrm{C} 4-\mathrm{C} 9 \\
& \mathrm{C} 3-\mathrm{C} 4-\mathrm{C} 9 \\
& \mathrm{C} 6-\mathrm{C} 5-\mathrm{C} 4 \\
& \mathrm{C} 6-\mathrm{C} 5-\mathrm{H} 5 \\
& \mathrm{C} 4-\mathrm{C} 5-\mathrm{H} 5 \\
& \mathrm{C} 5-\mathrm{C} 6-\mathrm{C} 7 \\
& \mathrm{C} 5-\mathrm{C} 6-\mathrm{C} 11 \\
& \mathrm{C} 7-\mathrm{C} 6-\mathrm{C} 11 \\
& \mathrm{O} 2-\mathrm{C} 7-\mathrm{C} 8 \\
& \mathrm{O} 2-\mathrm{C} 7-\mathrm{C} 6 \\
& \mathrm{C} 8-\mathrm{C} 7-\mathrm{C} 6 \\
& \mathrm{C} 7-\mathrm{C} 8-\mathrm{C} 9 \\
& \mathrm{C} 7-\mathrm{C} 8-\mathrm{H} 8 \\
& \mathrm{C} 9-\mathrm{C} 8-\mathrm{H} 8 \\
& \mathrm{C} 8-\mathrm{C} 9-\mathrm{C} 10 \\
& \mathrm{C} 8-\mathrm{C} 9-\mathrm{C} 4 \\
& \mathrm{C} 10-\mathrm{C} 9-\mathrm{C} 4 \\
& \mathrm{C} 1-\mathrm{C} 10-\mathrm{C} 9 \\
& \mathrm{C} 1-\mathrm{C} 10-\mathrm{H} 10 \\
& \mathrm{C} 9-\mathrm{C} 10-\mathrm{H} 10 \\
& \mathrm{O} 3-\mathrm{C} 11-\mathrm{C} 12 \\
& \mathrm{C} 18-\mathrm{O} 1-\mathrm{C} 1-\mathrm{C} 10 \\
& \mathrm{C} 18-\mathrm{O} 1-\mathrm{C} 1-\mathrm{C} 2 \\
& \mathrm{O} 1-\mathrm{C} 1-\mathrm{C} 2-\mathrm{C} 3 \\
& \mathrm{C} 10-\mathrm{C} 1-\mathrm{C} 2-\mathrm{C} 3 \\
& \mathrm{C} 1-\mathrm{C} 2-\mathrm{C} 3-\mathrm{C} 4 \\
& \mathrm{C} 2-\mathrm{C} 3-\mathrm{C} 4-\mathrm{C} 5 \\
& \mathrm{C} 2-\mathrm{C} 3-\mathrm{C} 4-\mathrm{C} 9 \\
& \mathrm{C} 3-\mathrm{C} 4-\mathrm{C} 5-\mathrm{C} 6 \\
& \mathrm{C} 9-\mathrm{C} 4-\mathrm{C} 5-\mathrm{C} 6 \\
& \mathrm{C} 4-\mathrm{C} 5-\mathrm{C} 6-\mathrm{C} 7 \\
& \mathrm{C} 4-\mathrm{C} 5-\mathrm{C} 6-\mathrm{C} 11 \\
& \mathrm{C} 19-\mathrm{O} 2-\mathrm{C} 7-\mathrm{C} 8 \\
& \mathrm{C} 19-\mathrm{O} 2-\mathrm{C} 7-\mathrm{C} 6 \\
& \mathrm{C} 5-\mathrm{C} 6-\mathrm{C} 7-\mathrm{O} 2 \\
& \mathrm{C} 11-\mathrm{C} 6-\mathrm{C} 7-\mathrm{C} 2 \\
& \mathrm{C} 5-\mathrm{C} 4-\mathrm{C} 9-\mathrm{C} 9-\mathrm{C} 10 \\
& \mathrm{C} 5-\mathrm{C} 6-\mathrm{C} 7-\mathrm{C} 8 \\
& \mathrm{C} 11-\mathrm{C} 6-\mathrm{C} 7-\mathrm{C} 8 \\
& \mathrm{O} 2-\mathrm{C} 7-\mathrm{C} 8-\mathrm{C} 9 \\
& \mathrm{C} 6-\mathrm{C} 7-\mathrm{C} 8-\mathrm{C} 9 \\
& \mathrm{C} 7-\mathrm{C} 8-\mathrm{C} 9-\mathrm{C} 10 \\
& \mathrm{C} 7-\mathrm{C} 4 \\
&
\end{aligned}
$$

119.4

$122.78(18)$

$118.58(16)$

$118.61(17)$

$122.20(17)$

118.9

118.9

$118.89(16)$

$118.06(17)$

$122.98(16)$

$124.72(16)$

$115.05(15)$

$120.19(16)$

$121.22(17)$

119.4

119.4

$121.58(18)$

$118.88(16)$

$119.50(17)$

119.90 (19)

120.1

120.1

120.38 (17)

$6.0(3)$

$-173.88(17)$

$179.56(18)$

$-0.3(3)$

$-0.2(3)$

-177.87 (19)

$0.2(3)$

$177.68(18)$

$-0.3(3)$

-0.9 (3)

-178.08 (17)

$-4.8(2)$

$172.93(15)$

$-176.14(16)$

0.9 (2)

1.7 (3)

$178.79(16)$

$176.31(16)$

$-1.4(3)$

$-177.62(16)$

$0.1(3)$

$0.8(3)$

$-177.36(17)$

$178.52(16)$
C13-C14-H14

$\mathrm{C} 14-\mathrm{C} 15-\mathrm{C} 16$

$\mathrm{C} 14-\mathrm{C} 15-\mathrm{Br} 1$

$\mathrm{C} 16-\mathrm{C} 15-\mathrm{Br} 1$

$\mathrm{C} 17-\mathrm{C} 16-\mathrm{C} 15$

C17-C16-H16

C15-C16-H16

$\mathrm{C} 16-\mathrm{C} 17-\mathrm{C} 12$

C16-C17-H17

C12-C17-H17

$\mathrm{O} 1-\mathrm{C} 18-\mathrm{H} 18 \mathrm{~A}$

O1-C18-H18B

$\mathrm{H} 18 \mathrm{~A}-\mathrm{C} 18-\mathrm{H} 18 \mathrm{~B}$

O1-C18-H18C

$\mathrm{H} 18 \mathrm{~A}-\mathrm{C} 18-\mathrm{H} 18 \mathrm{C}$

H18B-C18-H18C

O2-C19-H19A

O2-C19-H19B

H19A-C19-H19B

O2-C19- $\mathrm{H} 19 \mathrm{C}$

H19A-C19-H19C

$\mathrm{H} 19 \mathrm{~B}-\mathrm{C} 19-\mathrm{H} 19 \mathrm{C}$

$\mathrm{C} 3-\mathrm{C} 4-\mathrm{C} 9-\mathrm{C} 10$
$\mathrm{O} 1-\mathrm{C} 1-\mathrm{C} 10-\mathrm{C} 9$
$\mathrm{C} 2-\mathrm{C} 1-\mathrm{C} 10-\mathrm{C} 9$
$\mathrm{C} 8-\mathrm{C} 9-\mathrm{C} 10-\mathrm{C} 1$
$\mathrm{C} 4-\mathrm{C} 9-\mathrm{C} 10-\mathrm{C} 1$
$\mathrm{C} 5-\mathrm{C} 6-\mathrm{C} 11-\mathrm{O} 3$
$\mathrm{C} 7-\mathrm{C} 6-\mathrm{C} 11-\mathrm{O} 3$
$\mathrm{C} 5-\mathrm{C} 6-\mathrm{C} 11-\mathrm{C} 12$
$\mathrm{C} 7-\mathrm{C} 6-\mathrm{C} 11-\mathrm{C} 12$
$\mathrm{O} 3-\mathrm{C} 11-\mathrm{C} 12-\mathrm{C} 13$
$\mathrm{C} 6-\mathrm{C} 11-\mathrm{C} 12-\mathrm{C} 13$
$\mathrm{O} 3-\mathrm{C} 11-\mathrm{C} 12-\mathrm{C} 17$
$\mathrm{C} 6-\mathrm{C} 11-\mathrm{C} 12-\mathrm{C} 17$
$\mathrm{C} 17-\mathrm{C} 12-\mathrm{C} 13-\mathrm{C} 14$
$\mathrm{C} 11-\mathrm{C} 12-\mathrm{C} 13-\mathrm{C} 14$
$\mathrm{C} 12-\mathrm{C} 13-\mathrm{C} 14-\mathrm{C} 15$
$\mathrm{C} 13-\mathrm{C} 14-\mathrm{C} 15-\mathrm{C} 16$
$\mathrm{C} 13-\mathrm{C} 14-\mathrm{C} 15-\mathrm{B} 1$
$\mathrm{C} 14-\mathrm{C} 15-\mathrm{C} 16-\mathrm{C} 17$
$\mathrm{Br} 1-\mathrm{C} 15-\mathrm{C} 16-\mathrm{C} 17$
$\mathrm{C} 15-\mathrm{C} 16-\mathrm{C} 17-\mathrm{C} 12$
$\mathrm{C} 13-\mathrm{C} 12-\mathrm{C} 17-\mathrm{C} 16$
$\mathrm{C} 11-\mathrm{C} 12-\mathrm{C} 17-\mathrm{C} 16$

$0.4(3)$

$-178.98(17)$

0.9 (3)

$176.76(17)$

-0.9 (2)

46.0 (3)

$-131.1(2)$

$-132.63(18)$

$50.3(2)$

-154.64 (19)

24.0 (3)

24.1 (3)

-157.30 (19)

1.1 (3)

179.87 (18)

$1.2(3)$

$-2.7(3)$

$176.93(15)$

1.8 (3)

$-177.86(15)$

0.7 (3)

-2.1 (3)

179.15 (19) 
supporting information

Hydrogen-bond geometry $\left(\AA,{ }^{\circ}\right)$

\begin{tabular}{lllll}
\hline$D-\mathrm{H} \cdots A$ & $D-\mathrm{H}$ & $\mathrm{H} \cdots A$ & $D \cdots A$ & $D-\mathrm{H} \cdots A$ \\
\hline $\mathrm{C} 19-\mathrm{H} 19 A \cdots \mathrm{O} 1^{\mathrm{i}}$ & 0.96 & 2.53 & $3.477(2)$ & 170 \\
$\mathrm{C} 5-\mathrm{H} 5 \cdots \mathrm{Br}^{\mathrm{ii}}$ & 0.93 & 2.98 & $3.8441(18)$ & 155 \\
\hline
\end{tabular}

Symmetry codes: (i) $x+1, y, z+1$; (ii) $-x,-y,-z$. 